\title{
Ultra-High to Ultra-Low Drug-Loaded Micelles: Probing Host-Guest Interactions by Fluorescence Spectroscopy
}

\author{
Michael M. Lübtow, ${ }^{[a]}$ Henning Marciniak, ${ }^{[b]}$ Alexander Schmiedel, ${ }^{[b]}$ Markus Roos, $^{[b]}$ \\ Christoph Lambert, ${ }^{[b]}$ and Robert Luxenhofer*[a]
}

\begin{abstract}
Polymer micelles are an attractive means to solubilize water insoluble compounds such as drugs. Drug loading, formulations stability and control over drug release are crucial factors for drug-loaded polymer micelles. The interactions between the polymeric host and the guest molecules are considered critical to control these factors but typically barely understood. Here, we compare two isomeric polymer micelles, one of which enables ultra-high curcumin loading exceeding $50 \mathrm{wt} . \%$, while the other allows a drug loading of
\end{abstract}

only $25 \mathrm{wt} . \%$. In the low capacity micelles, steady-state fluorescence revealed a very unusual feature of curcumin fluorescence, a high energy emission at $510 \mathrm{~nm}$. Time-resolved fluorescence upconversion showed that the fluorescence life time of the corresponding species is too short in the highcapacity micelles, preventing an observable emission in steady-state. Therefore, contrary to common perception, stronger interactions between host and guest can be detrimental to the drug loading in polymer micelles.

\section{Introduction}

Curcumin (CUR) is a yellow, natural phenolic compound, which can be isolated from the rhizome of curcuma longa (turmeric). ${ }^{[1]}$ Besides its use as a popular spice and food supplement in major parts of the world, it regained significant scientific attention due to its various biological effects reported in recent years. ${ }^{[2]}$ These include antioxidant, ${ }^{[3]}$ cardioprotective ${ }^{[4]}$ neuroprotective $^{[5]}$ antidiabetic, ${ }^{[6]}$ anti-inflammatory ${ }^{[7]}$ and even antitumor $^{[8]}$ activities, which are presumably elicited by modulating various signaling molecules including interleukin- $1,{ }^{\left[{ }^{[0]}\right.} \mathrm{NF}-\mathrm{KB}^{[10]}$ and many more. ${ }^{[11]}$ This versatility is most likely due to the chemical reactivity of CUR (making it a non-discriminating pharmaceutically active component), as well as its instability, ${ }_{1}^{[12]}$ as its degradation products display biological properties as well. ${ }^{[13]}$ However, in combination with its intense color, CUR is also considered a so-called pan-assay interference compound (PAIN) or invalid metabolic panacea (IMP), making it appear to

[a] M. M. Lübtow, Prof. Dr. R. Luxenhofer

Functional Polymer Materials, Chair for Advanced Materials Synthesis Department of Chemistry and Pharmacy and Bavarian Polymer Institute University of Würzburg, Röntgenring 11, 97070 Würzburg (Germany) E-mail: robert.luxenhofer@uni-wuerzburg.de

[b] Dr. H. Marciniak, A. Schmiedel, M. Roos, Prof. Dr. C. Lambert Institute of Organic Chemistry and Center for Nanosystems Chemistry University of Würzburg, Am Hubland, 97070 Würzburg (Germany)

$\square$ Supporting information and the ORCID identification number(s) for the

(iD) author(s) of this article can be found under: https://doi.org/10.1002/chem.201902619.

of (c) 2019 The Authors. Published by Wiley-VCH Verlag GmbH \& Co. KGaA This is an open access article under the terms of Creative Commons Attribution NonCommercial-NoDerivs License, which permits use and distribution in any medium, provided the original work is properly cited, the use is non-commercial and no modifications or adaptations are made. be active even if it is not. ${ }^{[14]}$ Apart from this ongoing debate, ${ }^{[15]}$ the instability and extremely low water solubility $(\log P=$ $3.28,{ }^{[16]}$ solubility $\left.\left(\mathrm{H}_{2} \mathrm{O}\right)=0.6 \mathrm{mg} \mathrm{L}^{-1[17]}\right)$ result in a formidable challenge to formulate and safely deliver $\mathrm{CUR}^{,{ }^{[18]}}$ making it arguably an ideal model to test and challenge drug delivery systems. In recent decades, nanoformulations-that is, drugloaded particles or micelles in the nanosize range-have been investigated as promising drug delivery vehicles for numerous drugs, including CUR. ${ }^{[19]}$ Although being able to increase CUR water solubility, most of such nano-systems suffer rather low overall drug-loadings below $20 \mathrm{wt.} \%{ }^{[20]}$ and overall CUR solubility typically remains low. In the last few years, considerable efforts have been made to understand and utilize specific host-guest interactions such as donor-acceptor interactions, ${ }^{[21]}$ $\mathrm{H}$-bonding, ${ }^{[22]}$ complexation $^{[23]}$ and $\pi-\pi$ interactions $^{[24]}$ to improve drug loading and formulations stability. A simple poly(2oxazoline) $(\mathrm{POx})^{[25]}$ based micellar drug delivery system was previously reported featuring unexpected high drug loadings up to almost $50 \mathrm{wt} . \%$ paclitaxel (PTX) in combination with very high aqueous concentrations of $>40 \mathrm{gL}^{-1} \cdot{ }^{[26]}$ Using this ABAtriblock copolymer with two hydrophilic poly(2-methyl-2-oxazoline) (pMeOx) blocks $A$ and a modest hydrophobic poly(2-nbutyl-2-oxazoline) (pBuOx) block $\mathbf{B}=\mathbf{A}-\mathbf{p B u O x}-\mathbf{A}$ for the encapsulation of CUR, drug loadings up to 25 wt.\% could be achieved. ${ }^{[27]}$ However, a structural isomer of A-pBuOx-A with the same hydrophilic pMeOx shell, but a barely hydrophobic poly(2-n-propyl-2-oxazine) (pPrOzi) core $=\mathbf{A}$-pPrOzi-A enabled extremely high drug loadings up to $54 \mathrm{wt} . \%$ with a corresponding CUR solubility of $55 \mathrm{~g} \mathrm{~L}^{-1}$ (approximately $10^{5}$ fold increase in water solubility) while PTX loading was mediocre. ${ }^{[28]}$ Therefore, a simple formal shift of a methylene group from the polymer side chain (BuOx) to the polymer main chain (PrOzi) significantly affected drug compatibilities. Despite detailed mi- 
cellar analysis using for example, small angle neutron scattering, ${ }^{[29]}$ we were unable to describe the polymer-drug interactions that explain the ultra-high drug loading and the unexpected specificities on a molecular basis. ${ }^{[28,30]}$ Therefore, we wondered if we could get a better understanding of the ApBuOx-A/CUR and A-pPrOzi-A/CUR formulations by taking a closer look at the very inside of those drug-loaded micelles, namely the drug itself. CUR is highly solvatochromic, that is, its absorption and emission properties strongly depend on its microenvironment $^{[31]}$ causing Stokes shifts between 2000 and $6000 \mathrm{~cm}^{-1}$ in organic media (Figure 1). ${ }^{[32]}$ While in aprotic solvents, CUR lacks any specific interactions, hydrogen-bonding is present in protic solvents. ${ }^{[33]}$ Furthermore, the bis- $\alpha, \beta$-unsatu- ties to enable efficacious therapy. Insights into the interactions between polymer and drug gained from this study may help to improve our understanding of drug delivery systems and eventually improve their design. The relevance of such improved understanding will not be limited to the case of CUR. After all, the molecular interactions, that is, H-bonding, dipoledipole and Van der Waals interactions will be relevant for most drug delivery systems where the drug is solubilized by physical interactions. However, even if drugs are covalently bound to carrier molecules, these non-covalent interactions must be expected to influence the stability and morphology of drug delivery systems.

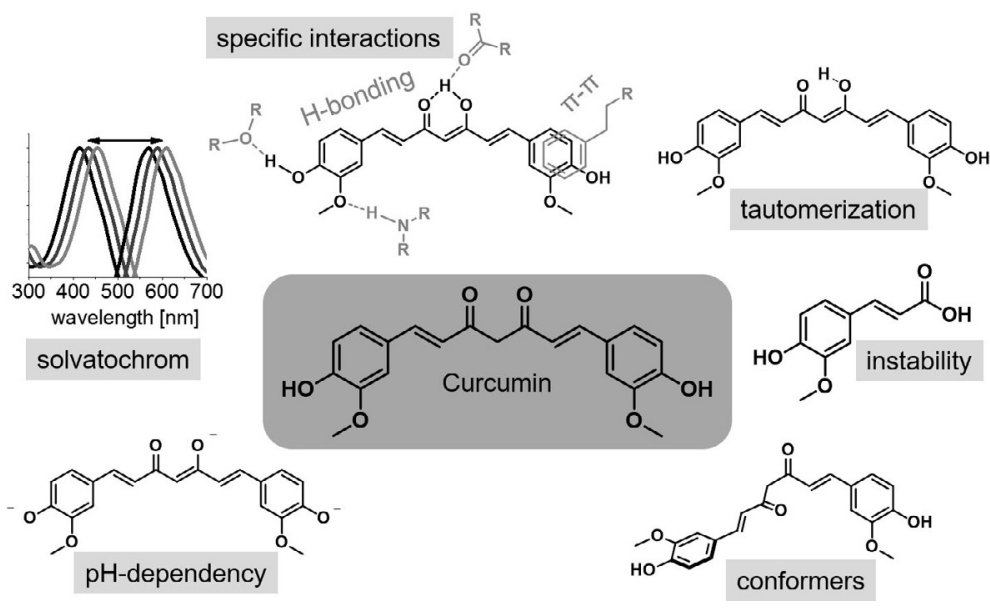

Figure 1. Scheme of the various influences that affect the optical properties of CUR including chemical stability, ${ }^{[12 a, b, 13 a]}$ solvatochromicity, ${ }_{1}^{[31 a, b, 33]}$ tautomerization, ${ }^{[34 a, b, 35,37,38,42,43]}$ $\mathrm{pH}^{[18,44]}$ conformers, ${ }^{[32,34,45]}$ as well as specific interactions. ${ }^{[33,46,47]}$

rated $\beta$-diketone exhibits keto-enol tautomerism with three main tautomers, each of them with various isomers. ${ }^{[34]}$ Stabilization of a certain tautomer can be facilitated by addition of surfactants, as shown by the stabilization of the keto-enol tautomer in the presence of anionic ${ }^{[35]}$ or zwitterionic ${ }^{[36]}$ surfac- $^{-}$ tants, as well as non-ionic micelles ${ }^{[37]}$ or nanodiscs. ${ }^{[38]}$ The effects of the microenvironment on CUR fluorescence in polymer micelles were previously investigated. The stronger the interference of the surrounding surfactant with non-radiative relaxation processes, the longer relaxation times were observed hinting towards stronger intermolecular interactions. ${ }^{[39]}$ Such dependency of the photorelaxation on the surrounding media is generally well established and has been first described by Schmidt in 1896. With increasing viscosity, internal conversion processes are suppressed. Therefore, fluorescence quantum yields strongly increase. ${ }^{[40]}$

Having these observations in mind, we wondered if the photophysical properties of CUR could help us to understand the different compatibilities and loading capacities of A-pBuOx-A and A-pPrOzi-A. As in modern drug-discovery programs a shift towards ever more hydrophobic compounds with poor water solubility is observed ${ }^{[41]}$ a good compatibility between carrier and drug is necessary to achieve sufficiently high drug solubili-

\section{Experimental Section}

\section{Reagents}

The polymers A-pBuOx-A (Me- $\mathrm{MeOx}_{35}-\mathrm{BuOx}_{20}-\mathrm{MeOx}_{35}-$ Pip) and A-pPrOzi-A (Me-MeOx ${ }_{35}-\mathrm{PrOzi}_{20}-\mathrm{MeOx}_{35}$-PipBoc) were synthesized and described previously. ${ }^{[27]}$ Curcumin powder from Curcuma longa (turmeric) was purchased from Sigma-Aldrich and analyzed in-house (curcumin = $79 \%$; demethoxycurcumin $=17 \%$, bisdemethoxycurcu$\min =4 \%$; determined by HPLC analysis; no difference in fluorescence upconversion experiments between this curcuminoid mixture and pure CUR (>98\%) were observed by Petrich and co-workers $\left.{ }^{[48]}\right)$.

\section{Curcumin encapsulation}

Curcumin-loaded polymer micelles were prepared by the thin film method. ${ }^{[26 a]}$ Ethanolic polymer $\left(20 \mathrm{~g} \mathrm{~L}^{-1}\right)$ and curcumin $\left(5.0 \mathrm{~g} \mathrm{~L}^{-1}\right)$ stock solutions were mixed in desired ratio. After complete removal of the solvent at $55^{\circ} \mathrm{C}$ under a mild stream of argon, the films were dried in vacuo $(\leq 0.2 \mathrm{mbar})$ for at least $20 \mathrm{~min}$. Subsequently, preheated $\left(37^{\circ} \mathrm{C}\right) \mathrm{H}_{2} \mathrm{O}$ (Millipore) was added to obtain final polymer and curcumin concentrations as mentioned in the main text. To ensure complete solubilization, the solutions were shaken at $55^{\circ} \mathrm{C}$ for $15 \mathrm{~min}$ at $1250 \mathrm{rpm}$ with a Thermomixer comfort (Eppendorf AG, Hamburg, Germany). Non-solubilized curcumin (if any) was removed by centrifugation for $5 \mathrm{~min}$ at $9000 \mathrm{rpm}$ with a MIKRO 185 (Hettich, Tuttlingen, Germany). Curcumin quantification was performed by UV/Vis absorption of diluted samples in ethanol using a BioTek Eon Microplate Spectrophotometer (Thermo Fisher Scientific, MA, USA) using a calibration curve obtained with known amounts of CUR. ${ }^{[27]}$

\section{Absorption}

Absorption spectra at room temperature were recorded from 200 $800 \mathrm{~nm}$ with a Cary 50 UV/Vis Spectrophotometer (Agilent, Waldbronn, Germany). Neat CUR was dispersed in $\mathrm{H}_{2} \mathrm{O}\left(1 \mathrm{mg} \mathrm{L}^{-1}\right.$; $2.7 \mu \mathrm{M}$; undissolved particles were removed by centrifugation) and measured in quartz cuvettes $(d=10 \mathrm{~mm}, \mathrm{QS}$, Hellma, Müllheim, Germany). CUR encapsulated into polymer micelles was measured undiluted (polymer $=10 \mathrm{~g} \mathrm{~L}^{-1}, \mathrm{CUR}=0.05-12 \mathrm{~g} \mathrm{~L}^{-1}$ ) in quartz cuvettes $(d=0.01 \mathrm{~mm})$.

All temperature-dependent measurements were performed in quartz cuvettes $(d=10 \mathrm{~mm})$ with a Cary 5000 UV/Vis-NIR (Agilent) equipped with a Cary Dual Cell Peltier Accessory (Agilent) from $235 \mathrm{~nm}$ to $800 \mathrm{~nm}$ (bandwidth $=2 \mathrm{~nm}$ ) at $600 \mathrm{~nm} \mathrm{~min}^{-1}$. Neat CUR 
dissolved in $\mathrm{H}_{2} \mathrm{O}\left(\approx 1 \mathrm{mg} \mathrm{L}^{-1}\right)$ was heated from $15^{\circ} \mathrm{C}$ to $80^{\circ} \mathrm{C}$ (steps of $5^{\circ} \mathrm{C}$ ) and cooled from $80^{\circ} \mathrm{C}$ to $15^{\circ} \mathrm{C}$ (steps of $10^{\circ} \mathrm{C}$ ). Stock solutions of polymer-formulated CUR ([CUR] $=0.1-12 \mathrm{~g} \mathrm{~L}^{-1}$ ) were diluted to [CUR] $\approx 0.01 \mathrm{gL}^{-1}(27 \mu \mathrm{M})$ and heated from $20^{\circ} \mathrm{C}$ to $60^{\circ} \mathrm{C}$ (steps of $5^{\circ} \mathrm{C}$ ) and cooled from $60^{\circ} \mathrm{C}$ to $20^{\circ} \mathrm{C}$ (steps of $10^{\circ} \mathrm{C}$ ). At each temperature, the samples were allowed to equilibrate for $10 \mathrm{~min}$.

\section{Steady-state fluorescence}

Steady-state emission $\left(\lambda_{\mathrm{ex}}=420 \mathrm{~nm} ; \lambda_{\mathrm{em}}=430 \mathrm{~nm}-700 \mathrm{~nm}\right)$ and excitation $\left(\lambda_{\mathrm{em}}=540 \mathrm{~nm} ; \lambda_{\mathrm{ex}}=300 \mathrm{~nm}-530 \mathrm{~nm}\right)$ spectra were recorded in quartz cuvettes $(d=10 \mathrm{~mm})$ with a Spectrofluorometer FP8300 (JASCO, Pfungstadt, Germany) equipped with a F250 recirculating chiller (Julabo, Seelbach, Germany) at a response of $0.1 \mathrm{sec}$ and a scan speed of $1000 \mathrm{nmmin}^{-1}$ (Xe lamp, ex and em bandwidth: $2.5 \mathrm{~nm}$ ) at $25^{\circ} \mathrm{C}$. Spectra are presented as the average of 3 accumulated measurements of the same sample. Stock solutions of polymer-formulated CUR ([CUR] $=0.05-12 \mathrm{~g} \mathrm{~L}^{-1}$, [polymer] $=$ $10 \mathrm{~g} \mathrm{~L}^{-1}$ ) were diluted prior to the measurements to [CUR] $=$ $0.05 \mathrm{~g} \mathrm{~L}^{-1}(136 \mu \mathrm{M})$. For A-pPrOzi-A/CUR, detector sensitivity was set to "high". Due to the stronger fluorescence, detector sensitivity was set to "medium" in the case of A-pBuOx-A/CUR. A comparison of the CUR fluorescence at identical instrument settings is given in the supporting information (Figure S5). Fluorescence spectra were smoothed using OriginPro 2015G.

For temperature dependent measurements, neat CUR dissolved in $\mathrm{H}_{2} \mathrm{O}\left(\approx 1 \mathrm{mg} \mathrm{L}^{-1}\right)$ was heated from $15^{\circ} \mathrm{C}$ to $80^{\circ} \mathrm{C}$ (steps of $5^{\circ} \mathrm{C}$ ) and re-cooled from $80^{\circ} \mathrm{C}$ to $15^{\circ} \mathrm{C}$ (steps of $10^{\circ} \mathrm{C}$ ). Stock solutions of polymer-formulated CUR ([CUR] $=0.1-12 \mathrm{~g} \mathrm{~L}^{-1}$ ) were diluted to [CUR] $\approx 0.01 \mathrm{~g} \mathrm{~L}^{-1}(27 \mu \mathrm{M})$ and heated from $20^{\circ} \mathrm{C}$ to $60^{\circ} \mathrm{C}$ (steps of $5^{\circ} \mathrm{C}$ ) and re-cooled from $60^{\circ} \mathrm{C}$ to $20^{\circ} \mathrm{C}$ (steps of $10^{\circ} \mathrm{C}$ ). At each temperature, the samples were allowed to equilibrate for $10 \mathrm{~min}$.

\section{Fluorescence upconversion studies}

We used a commercial broadband fluorescence upconversion setup (FLUPS) ${ }^{[49]}$ from LIOPTEC which can simultaneously measure $395-850 \mathrm{~nm}$ fluorescence, with an intrinsic resolution of $0.9 \mathrm{~nm}$ (303-516 nm upconverted, intrinsic resolution $0.42 \mathrm{~nm}$ ). The fluorescence is focused onto a BBO crystal (BBO type II with a thickness of $100 \mu \mathrm{m}$, theta $=40^{\circ}$ and $\mathrm{phi}=0^{\circ}$ ) where it temporally and spatially overlaps with a gate pulse. The pump pulse, which was used to excite the sample, was guided over a delay stage to provide the temporal shift between gate pulse and fluorescence signal. Except of two lenses all optics were reflective to prevent pulse broadening.

The laser source was a chirped pulse amplification system "Solstice" from Newport-Spectra-Physics. This system provided a fundamental wavelength of $800 \mathrm{~nm}$ with $100 \mathrm{fs}$ pulse length and ca. $3.5 \mathrm{~mJ}$ pulse energy at a repetition rate of $1 \mathrm{kHz}$. To operate the FLUPS we split off about $0.75 \mathrm{~mJ}$.

To produce the gate pulse, we used a two-stage optical parametric amplifier (OPA) which was set to $\approx 1300 \mathrm{~nm}$. The pump energy for the OPA was around $0.25 \mathrm{~mJ}$ and the output energy was around $0.06 \mathrm{~mJ}$. Afterwards the gate pulse was guided onto the crystal through a compressor comprising only three instead of the usual four prisms. In order to shorten the instrument response function (ca. $150 \mathrm{fs}$ ) the 4 th prism was removed to retain the tilt of the wavefront and to match it with the wavefront from the fluorescence. The position where the 4 th prism is in a typical 4 prism compressor was projected by a lens onto the BBO crystal.
The $400 \mathrm{~nm}$ pump pulse was created by frequency doubling of the $800 \mathrm{~nm}$ fundamental and guided over a delay line with a maximum delay of $1.5 \mathrm{~ns}$ and an intrinsic resolution of $0.5 \mathrm{fs}$ to the sample (1 $\mathrm{mm}$ optical path length quartz cuvette).

The upconverted signal is guided over curved mirrors into a fiber coupled polychromator. To detect the signal a CCD-camera with full vertical binning and 2 pix horizontal binning was used (Andor iDus DV420A-BU, backside illuminated, $1024 \times 255$ Pixel, $26 \mu \mathrm{m} \times$ $26 \mu \mathrm{m})$.

The recorded fluorescence spectra were corrected for group velocity dispersion and photometric accuracy using a set of reference dyes with known spectral distribution.

\section{Anisotropy studies}

Polarized steady-state emission spectra $\left(\lambda_{\mathrm{ex}}=420 \mathrm{~nm} ; \lambda_{\mathrm{em}}=450 \mathrm{~nm}\right.$ $-800 \mathrm{~nm})$ were recorded in quartz cuvettes $(d=10 \mathrm{~mm})$ with a fluorescence lifetime spectrometer FLS980 (Edinburgh Instruments, Livingston, United Kingdom, software F980 version 1.2.2) equipped with a 450 W Xenon lamp, a PMT detector (R928P) and GlanThompson polarizers for excitation and emission at $25^{\circ} \mathrm{C}$. Measurements were performed as sequences with parallel and perpendicular orientation of the polarizers. Aqueous solutions were purged with argon for $10 \mathrm{~min}$ before the measurement.

\section{Addition of polymer to drug formulations}

Dynamics of CUR-loaded polymers were investigated by preparing aqueous stock solutions of A-pBuOx-A/CUR $=10 / 4 \mathrm{gL}^{-1}$ and ApPrOzi-A/CUR $=10 / 12 \mathrm{gL}^{-1}$. Subsequently, stock solutions were diluted $1 / 40(\mathrm{v} / \mathrm{v})$ with $\mathrm{H}_{2} \mathrm{O}$ and dry powder of the respective polymer was added to obtain the desired polymer/CUR ratios as mentioned in main text. For fluorescence measurements, all samples with added polymer were diluted to $[C U R]=0.03 \mathrm{~g} \mathrm{~L}^{-1}$.

\section{DLS measurements}

DLS measurements were performed on a Zetasizer Nano ZSP (Malvern Panalytical GmbH, Kassel, Germany) with a $633 \mathrm{~nm}$ HeNe-laser at $173^{\circ}$. Autocorrelations for each sample were obtained 3 times for 40 seconds and results are presented as mean. Prior to the measurements, all samples (polymer $=10 \mathrm{gL}^{-1}$ ) were diluted $1 / 10$ (v/v) with $\mathrm{H}_{2} \mathrm{O}$ to polymer $=1 \mathrm{~g} \mathrm{~L}^{-1}$ and filtered with $0.45 \mu \mathrm{M}$ PVDF syringe filters (Carl Roth $\mathrm{GmbH}+\mathrm{Co}$. KG, Karlsruhe, Germany). Selected samples were measured unfiltered to exclude any influence of the filtering on particle sizes $<1000 \mathrm{~nm}$. Samples were measured in quartz cuvettes ( $d=10 \mathrm{~mm}, \mathrm{QS}$, Hellma).

For temperature-dependent DLS measurements, the same regimen as for the photophysical studies was used. Briefly, the samples were heated from $20^{\circ} \mathrm{C}$ to $60^{\circ} \mathrm{C}$ (steps of $5^{\circ} \mathrm{C}$ ) and cooled from $60{ }^{\circ} \mathrm{C}$ to $20^{\circ} \mathrm{C}$ (steps of $10^{\circ} \mathrm{C}$ ). At each temperature, the samples were allowed to equilibrate for $10 \mathrm{~min}$.

\section{Results and Discussion}

When dissolved in $\mathrm{H}_{2} \mathrm{O}, \mathrm{CUR}$ exhibits two major absorption bands at $\lambda>300 \mathrm{~nm}$ (Figure S1a in the Supporting Information), attributed to a $\pi-\pi^{*}$ transition of the single feruloyl $\left(\lambda_{\text {abs }}=345 \mathrm{~nm}\right)$ and a $\pi-\pi^{*}$ transition of the two conjugated feruloyl chromophores $\left(\lambda_{\mathrm{abs}}=420 \mathrm{~nm}\right)$, as reported in literature. ${ }^{[36,50]}$ With increasing temperature, the diketo-form is favored $^{[43]}$ and the band at $\lambda_{\mathrm{abs}}=345 \mathrm{~nm}$ becomes more promi- 
nent (Figure S1 a). This was found to be partially reversible upon cooling. When excited at $\lambda_{\mathrm{ex}}=420 \mathrm{~nm}$, CUR exhibited a broad fluorescence emission with $\lambda_{\mathrm{em}, \max }=540 \mathrm{~nm}$ (Figure S1 b; corresponding excitation spectra at $\lambda_{\mathrm{em}}=540 \mathrm{~nm}$ are shown in Figure S1 c). With increasing temperature, the fluorescence intensity decreased and $\lambda_{\text {em,max }}$ shifted slightly from $556 \mathrm{~nm}$ $\left(20^{\circ} \mathrm{C}\right)$ to $551 \mathrm{~nm}\left(80^{\circ} \mathrm{C}\right)$ representing minor changes in the microenvironment of CUR. ${ }^{[33]}$ Similar to the absorbance, the fluorescence returned to approximately $80 \%$ of its initial intensity upon cooling. A $20 \%$ degradation of CUR heated to $80^{\circ} \mathrm{C}$ is surprisingly low (heating/cooling cycle took approximately $4 \mathrm{~h}$ ), as much more pronounced degradation even at room temperature is reported in the literature. ${ }^{[51]}$

When encapsulated in POx- and POzi-based micelles, major changes in the absorption properties of CUR were observed. It should be noted, that CUR in the POx/POzi micelles was found invariably amorphous, irrespective of the drug loading or the polymer employed. ${ }^{[2,30 a]}$ Irrespective whether CUR was solubilized by POx based A-pBuOx-A or the POzi based A-pPrOzi-A, the absorption at $\lambda_{\mathrm{abs}}=345 \mathrm{~nm}$ completely disappeared (Figure $2 \mathrm{a}, \mathrm{d})$ which may be attributed to hydrogen bonding between the keto-enol group of CUR ( $\mathrm{H}$-bond donor) and the carbonyl groups of the polymer (H-bond acceptor). ${ }^{[36]}$ The absorbance linearly increased with increasing CUR content until maximum drug-loading was achieved $\left(12 \mathrm{~g} \mathrm{~L}^{-1}\right.$ for A-pPrOzi-A; $4 \mathrm{gL}^{-1}$ for A-pBuOx-A). The corresponding molar extinction coefficient $\varepsilon$ of $8.5 \times 10^{4} \mathrm{dm}^{3} \mathrm{~mol}^{-1} \mathrm{~cm}^{-1} \quad\left(\varepsilon_{\mathrm{MeOH}}=6.8 \times 10^{4}\right.$; $\left.\varepsilon_{\mathrm{EtOH}}=5.5 \times 10^{4}\right)^{[52]}$ demonstrated the strong absorption of CUR in these aqueous formulations. A pronounced hypsochromic shift of $\lambda_{\text {abs,max }}$ from $432 \mathrm{~nm}$ ([CUR] $=0.05 \mathrm{~g} \mathrm{~L}^{-1}$ ) to $414 \mathrm{~nm}$ $\left([C U R]=12 \mathrm{~g} \mathrm{~L}^{-1}\right)$ ) was observed in the case of A-pPrOzi-A (Figure $2 \mathrm{~b}$ ), which is commonly attributed to a less polar microenvironment of CUR. ${ }^{[38,43]}$ We posit that particularly at low loading, the micellar core might still contain a certain amount of water, which becomes expelled as more CUR is incorporated. In accordance with this assumption, the size of A-pPrOzi-A/ CUR micelles which only form in the presence of CUR initially decreased with increasing CUR content ([CUR] $\leq 6 \mathrm{~g} \mathrm{~L}^{-1}$ ), before they increased in size (Figure $2 \mathrm{C}$ ), as reported previously. ${ }^{[2]} \mathrm{A}$ similar initial shrinkage was observed for A-pBuOx-A loaded with paclitaxel (PTX). ${ }^{[26 b, 29]}$ While $\lambda_{\text {abs,max }}$ at a certain CUR concentration was the same for both polymers (Figure $2 \mathrm{~b}, \mathrm{e}$ ), the size of the CUR-loaded micelles differed significantly. At $0.5 \mathrm{~g} \mathrm{~L}^{-1}$, only a single species with a hydrodynamic diameter $\left(D_{h}\right)$ of $14 \mathrm{~nm}$ was present in the case of A-pBuOx-A (Figure $2 \mathrm{f}$, Figure S3). However, with increasing CUR content, a second, much larger population occurred which became dominant at higher CUR-loadings. Hydrodynamic diameters between $550 \mathrm{~nm}\left([C U R]=1 \mathrm{~g} \mathrm{~L}^{-1}\right)$ and $120 \mathrm{~nm}\left([C U R]=4 \mathrm{~g} \mathrm{~L}^{-1}\right)$ suggested the presence of larger aggregates such as worm-like micelles or polymersomes and/or indicates colloidal instabilities which cause the A-pBuOx-A/CUR formulations to collapse at [CUR] $>4 \mathrm{gL}^{-1} \cdot{ }^{[27-28]}$ However, we would like to stress that these values should be considered with considerable care, as they were obtained using a rather simplistic equipment (Zetasizer Nano ZSP) observing only a single scattering angle. Also,
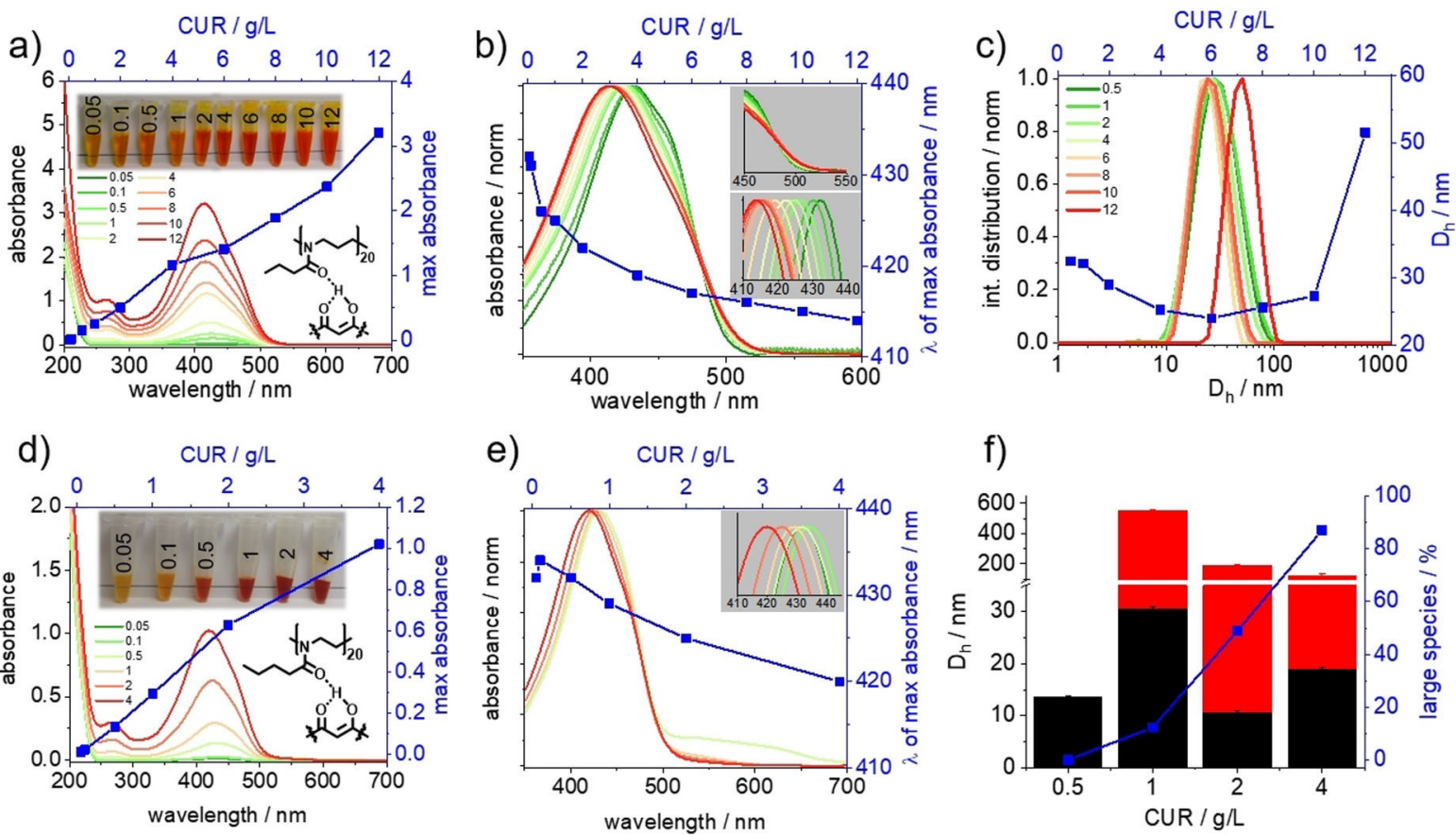

Figure 2. Absorption spectra and DLS measurements of A-pPrOzi-A/CUR (a,b,c) and A-pBuOx-A/CUR (d,e,f) formulations at a constant polymer concentration of $10 \mathrm{~g} \mathrm{~L}^{-1}$ and various CUR concentrations (legend in figures). Absorption spectra of A-pPrOzi-A/CUR (a) and A-pBuOx-A (d) with corresponding maximum absorbances (blue curve; top, right axis). Normalized absorption spectra of A-pPrOzi-A/CUR (b) and A-pBuOx-A/CUR (e) with corresponding $\lambda_{\text {max }}$ (blue curve; top, right axis). All absorption measurements were conducted undiluted in quartz cuvettes with $d=10 \mu \mathrm{m}$. c) Size distribution (intensity) of $\mathrm{A}-\mathrm{pPrOzi}-\mathrm{A} / \mathrm{CUR}$ formulations with corresponding $D_{\mathrm{h}}$ (blue curve; top, right axis). f) $D_{\mathrm{h}}$ of A-pBuOx-A/CUR formulations (black bars: smaller species; red bars: larger species; left axis) and corresponding percentage of the larger species (blue curve; right axis). Prior to the DLS measurements, the aqueous samples were diluted $1 / 10$ $(\mathrm{v} / \mathrm{v})$ with $\mathrm{H}_{2} \mathrm{O}$ to yield a polymer concentration of $1 \mathrm{~g} \mathrm{~L}^{-1}$. 
we should stress that the differences between the sizes and morphologies at higher drug loading are interesting, but most likely only to be attributed to differences in colloidal stabilities of the drug-loaded micelles. This phenomenon is currently under more detailed investigation but bears only little relevance to the subject matter of the current contribution, where we concentrate on the interactions of the micellar core and the incorporated molecules.

Fluorescence measurements were conducted after dilution to $[C U R]=0.05 \mathrm{gL}^{-1}(0.14 \mathrm{~mm})$ and varying corresponding polymer concentrations. For the sake of comparison with previous spectra, the samples are labeled according to the polymer/CUR (w/w; P/C) ratio, but the reader should bear in mind that the actual CUR concentration was the same in all samples. When excited at $\lambda_{\mathrm{ex}}=420 \mathrm{~nm}$, the fluorescence intensity decreased with increasing CUR loading, irrespective of the polymer (Figure $3 \mathrm{a}, \mathrm{c}$ ), which is attributed to self-quenching. At $\mathrm{P} /$ $C=10 / 0.1$, only approx. 0.2 CUR molecules per macromolecule (or approx. 20 hydrophobic repeat units) comprise the micelles. At $P / C=10 / 4$, already approx. nine CUR molecules per polymer chain are present, meaning per CUR molecule only two relatively small hydrophobic repeat units with their respective amides are available. Following this, at $P / C=10 / 12$, a staggering 27 CUR molecules per polymer chains are incorporated. At this point, the number of CUR molecules outnumbers hydrophobic repeat units in the polymers.

The wavelength of maximum emission $\left(\lambda_{\mathrm{em}, \max }\right)$ increased with increasing CUR content by approximately $15 \mathrm{~nm}$, irrespec- tive of the polymer (Figure $3 \mathrm{~b}, \mathrm{~d}$ ). This led to an increased Stokes shift from $5200 \mathrm{~cm}^{-1}(P / C=10 / 0.05)$ to $6600 \mathrm{~cm}^{-1}(P /$ $\mathrm{C}=10 / 12$ ) in the case of A-pPrOzi-A (Figure $\mathrm{S} 4 \mathrm{a}$ ) and from $5000 \mathrm{~cm}^{-1}(P / C=10 / 0.05)$ to $6000 \mathrm{~cm}^{-1}(P / C=10 / 4)$ in the case of A-pBuOx-A (Figure $\mathrm{S} 4 \mathrm{~b}$ ). In aprotic as well as protic solvents, the Stokes shift of CUR generally increases with increasing solvent polarity. ${ }^{[33,53]}$ In accordance with the LippertMataga equation, this was correlated to solvent polarity. In our case, especially at high CUR loadings, CUR itself would dominate the surrounding media, that is, most CUR molecules are surrounded by other CUR molecules with some polymer chains in between preventing crystallization. Steady-state fluorescence anisotropy studies showed a significant difference between the two micelle types. With increasing CUR content, CUR anisotropy $r_{0}$ decreased in both polymeric systems. However, while for A-pPrOzi-A/CUR $r_{0}$ decreased gradually, ApBuOx-A/CUR exhibited a steep decay from [CUR] $=0.05 \mathrm{gL}^{-1}$ to $1 \mathrm{~g} \mathrm{~L}^{-1}$ (Figure $\mathrm{S6}$ ). This steep decrease in anisotropy coincided well with the occurrence of the second, much larger aggregates observed by DLS (Figure $2 \mathrm{f}$ ). Such decrease in anisotropy is often attributed to an increased molecular flexibility of the fluorophore. However, in the present system, it seems more reasonable to assume energy transfer between different CUR molecules within the micelles, which is well known to depolarize fluorescence.

A closer look the shape of the emission spectra revealed two types of emissions for A-pBuOx-A/CUR (Figure $3 \mathrm{~d}$ ). Besides the emission at $\lambda_{\mathrm{em}} \approx 550 \mathrm{~nm}$, a second, higher energy
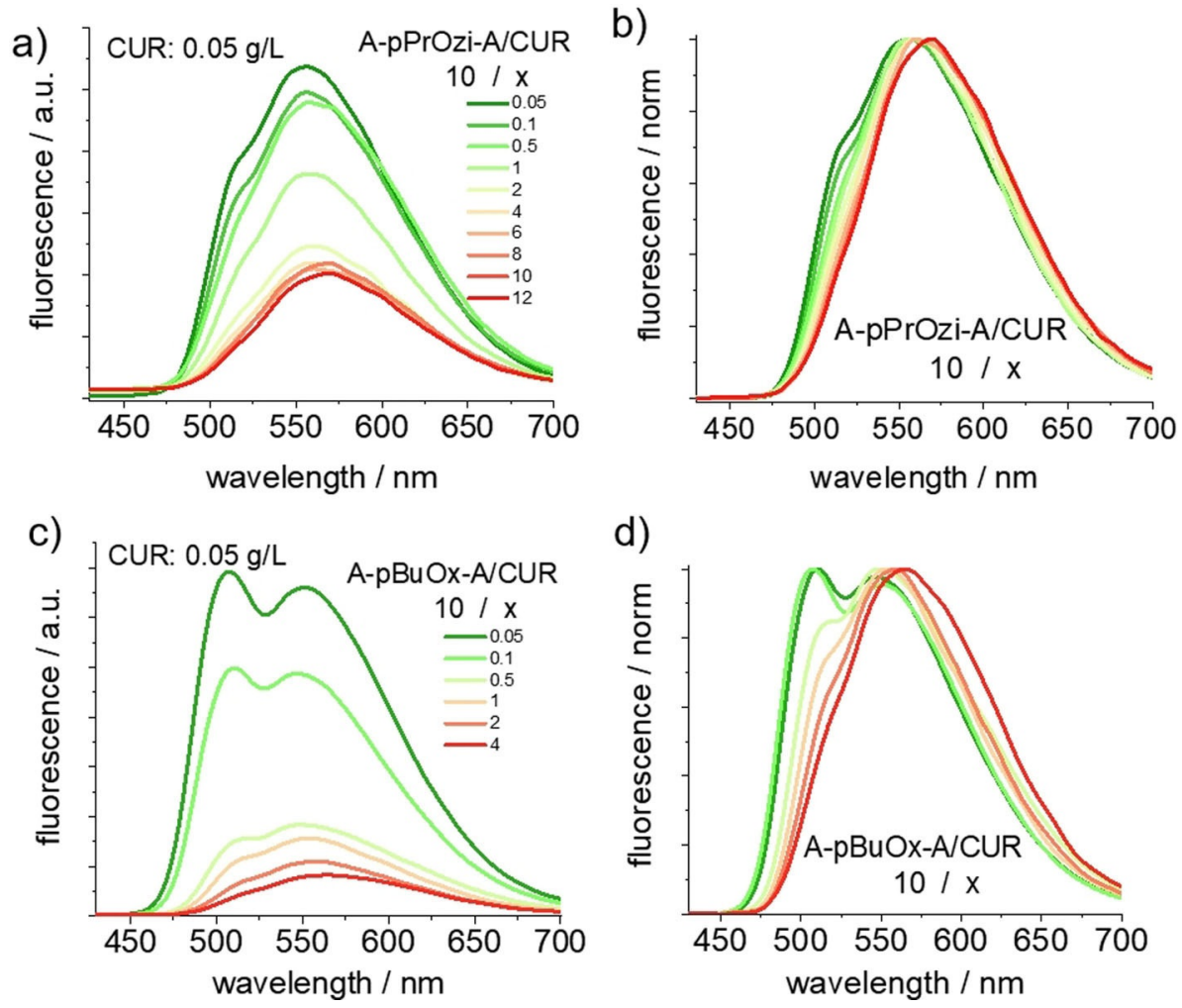

d)

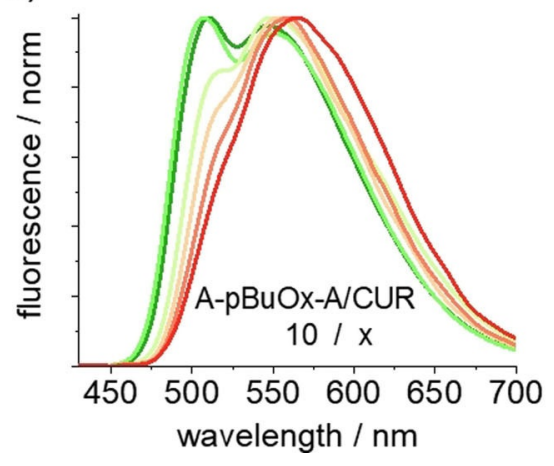

Figure 3. Fluorescence spectra of A-pPrOzi-A/CUR (a) and A-pBuOx-A/CUR (c) at $c(C U R)=0.05 \mathrm{gL}^{-1}$ and different polymer/CUR ratios, measured in quartz cuvettes with $d=10 \mathrm{~mm}$. Normalized emission spectra of A-pPrOzi-A/CUR (b) and A-pBuOx-A/CUR (d). 
emission at $\lambda_{\mathrm{em}} \approx 510 \mathrm{~nm}$ is visible. This emission became dominant at very low drug loadings ( $P / C \geq 10 / 0.1)$. In contrast, for A-pPrOzi-A, only a small shoulder appeared in this region (Figure $3 \mathrm{~b}$ ). The keto-enol form of CUR undergoes a fast intramolecular proton transfer in the excited state (ESIPT). ${ }^{[45]}$ One may be tempted to argue that one of the emissions at $\lambda_{\mathrm{em}}$ $\approx 550 \mathrm{~nm}$ and $510 \mathrm{~nm}$ derives from the emission of the proton-transferred isomer, and the other one from the nontransferred geometry. ${ }^{[54]}$ However, in that case energetically different $S_{1}$ and $S_{0}$ states of the different isomers are a prerequisite. Although otherwise stated in literature, ${ }^{[55]}$ this is not the case for CUR due to its highly symmetric structure. Banerjee et al. ${ }^{[56]}$ also observed two emission bands $\left(\lambda_{\text {em }}=461 \mathrm{~nm}\right.$ and $485 \mathrm{~nm}$ ) for CUR encapsulated in the polar core of reverse micelles dissolved in $n$-heptane. With increasing amount of polar solvents incorporated into the micellar core, the high-energy emission vanished. This was attributed to the transition of CUR from the bulk $n$-heptane phase $\left(\lambda_{\mathrm{em}}=461 \mathrm{~nm}\right)$ to the micellar core $\left(\lambda_{\mathrm{em}}=485 \mathrm{~nm}\right)$. However, in the present case, the concentration of CUR in the bulk phase water is negligible (water solubility of [CUR] $<1 \mathrm{mg} \mathrm{L}^{-1[17]}$. As we kept the absolute concentration of CUR the same in all samples $\left([C U R]=50 \mathrm{mg} \mathrm{L}^{-1}\right.$ ), the polymer concentration was the only significant variable. As the high-energy emission at $\lambda_{\mathrm{em}} \approx 510 \mathrm{~nm}$ occurred only at high $\mathrm{P} /$ $C$ ratios it seems reasonable that this emission band is due to specific polymer-CUR interactions with a corresponding stabilized $S_{1}$-state. At $P / C=10 / 0.05$, approximately 9 polymer chains or 170 repeat units of the hydrophobic core are present per CUR molecule. At such high P/C ratios, the interactions between CUR and the excess polymer should dominate and one can expect that individual CUR molecules only interact with the polymer repeat units, presumably via H-bonding. In contrast, at $P / C=10 / 4$, only 2 repeat units of the hydrophobic block are available per CUR molecule and interactions between individual CUR molecules appear to dominate the spectroscopic behavior. Most interestingly, A-pPrOzi-A, enabling much higher CUR-loadings than A-pBuOx-A, did only exhibit a small shoulder at the high-energy emission (Figure $3 \mathrm{~b}$ ). In addition, the measured fluorescence intensity of A-pBuOx-A/CUR was much higher compared to the fluorescence intensity measured in the A-pPrOzi-A/CUR system at P/C $>10 / 2$ (Figure S5).

Considering that the absorbance at identical CUR content is the same, the higher fluorescence intensity must be correlated with a higher fluorescence quantum yield. Such increase in fluorescence quantum yield of fluorophores is connected with a decrease of the rate of (non-emitting) internal conversion of excited states. ${ }^{[40 b]}$ This decrease in the rate of internal conversions may be attributed to an increase in the viscosity of the immediate environment, that is, the molecular mobility of the fluorophore. ${ }^{[40 a]}$ In the present case, this may be interpreted as measure of the interaction between the fluorophore and the host polymer in combination with the flexibility of the polymer chain surrounding the fluorophore. The inherent flexibility of pPrOzi is higher compared to that of pBuOx due to the additional methylene group in the polymer backbone. ${ }^{[57]}$ In the case of reverse micelles, the fluorescence intensity of CUR was increased by modulation of the non-radiative rates associated with excited-state intermolecular hydrogen bonding between CUR and polar solvents incorporated into the micellar core. ${ }^{[56]}$ Similarly, solvents that interact with the enol (acting as $\mathrm{H}$ donor) or with the ketone (acting as $\mathrm{H}$-acceptor) of CUR can interfere with internal conversion relaxation, for example, prolonging the fluorescence lifetime. ${ }^{[58]}$ To investigate this in more detail, fluorescence upconversion experiments were performed at low drug loading (10/0.5 and 10/0.05), as under these conditions considerable differences in the steady-state spectra were observed.

Here, we excited the samples at $400 \mathrm{~nm}$ and detected the time resolved emission spectra by focusing the fluorescence onto a BBO nonlinear optical crystal and by upconverting the fluorescence with a time delayed gate pulse at $1320 \mathrm{~nm}$. The resulting time resolved spectra were corrected for chirp and photometric intensity (Figure 4). For the analysis, we integrated spectral ranges at $488-526 \mathrm{~nm}$ and at $540-588 \mathrm{~nm}$ which correspond to the two fluorescence peaks observed in the steady state spectra (Figure 3). However, because the steady state fluorescence intensity is the integral of the intensity vs. time and depends on both, the fluorescence lifetime of the respective state as well as the transition probability between the excited and the ground-state, the time resolved spectra look different from the steady state spectra. Thus, for the analysis, the decay in the selected spectral regions was fitted by multiple exponential functions also considering the instrument response function (for fitting parameters, please see Table S1). Comparing the high (540-588 nm) and low (488-526 nm) wavelength emission ranges immediately reveals that all samples decay significantly faster at shorter wavelengths (see Figure $4 \mathrm{a}$ vs. $\mathrm{b}$ and the amplitude average lifetimes ${ }^{[59]}$ which are proportional to the steady state emission intensity in the last column of Table S1). In the time-resolved fluorescence spectra the different decay rates of different wavelength regions appears as a more or less continuous shift of the emission maximum, particularly at higher $P / C$ ratios (10/0.5) (Figure $4 d, f$ ). However, a closer look at the spectra at lower P/C ratios (10/ 0.05 ) shows that the peak maximum of the low wavelength region is in fact almost constant and it is the ratio of the two decay regions which produced this apparent shift. At both emission ranges, A-pBuOx-A based formulations exhibited longer fluorescence lifetimes than the corresponding ApPrOzi-A based CUR formulations (Figure $4 a, b$ and Table S1). The difference between the two polymers was more pronounced at low CUR loadings. For both polymers, the higher loaded micelles exhibit shorter lifetimes. Both observations are in accordance with the higher fluorescence intensities of the polymer/CUR formulations at low CUR loadings in steady state emission measurements (Figure $3 a, c$ ). The life time measurements also clearly show that the difference in steady state fluorescence between A-pPrOzi-A and A-pBuOx-A cannot be attributed to different species of the fluorophores, but only to different fluorescence lifetimes of the same species. The influence of the polymer/CUR ratio on fluorescence quantum yields was not further investigated, but the correlation of fluorescence quantum yields and local viscosities are well established. ${ }^{[40 a, c]}$ The high-energy emission can therefore be attribut- 

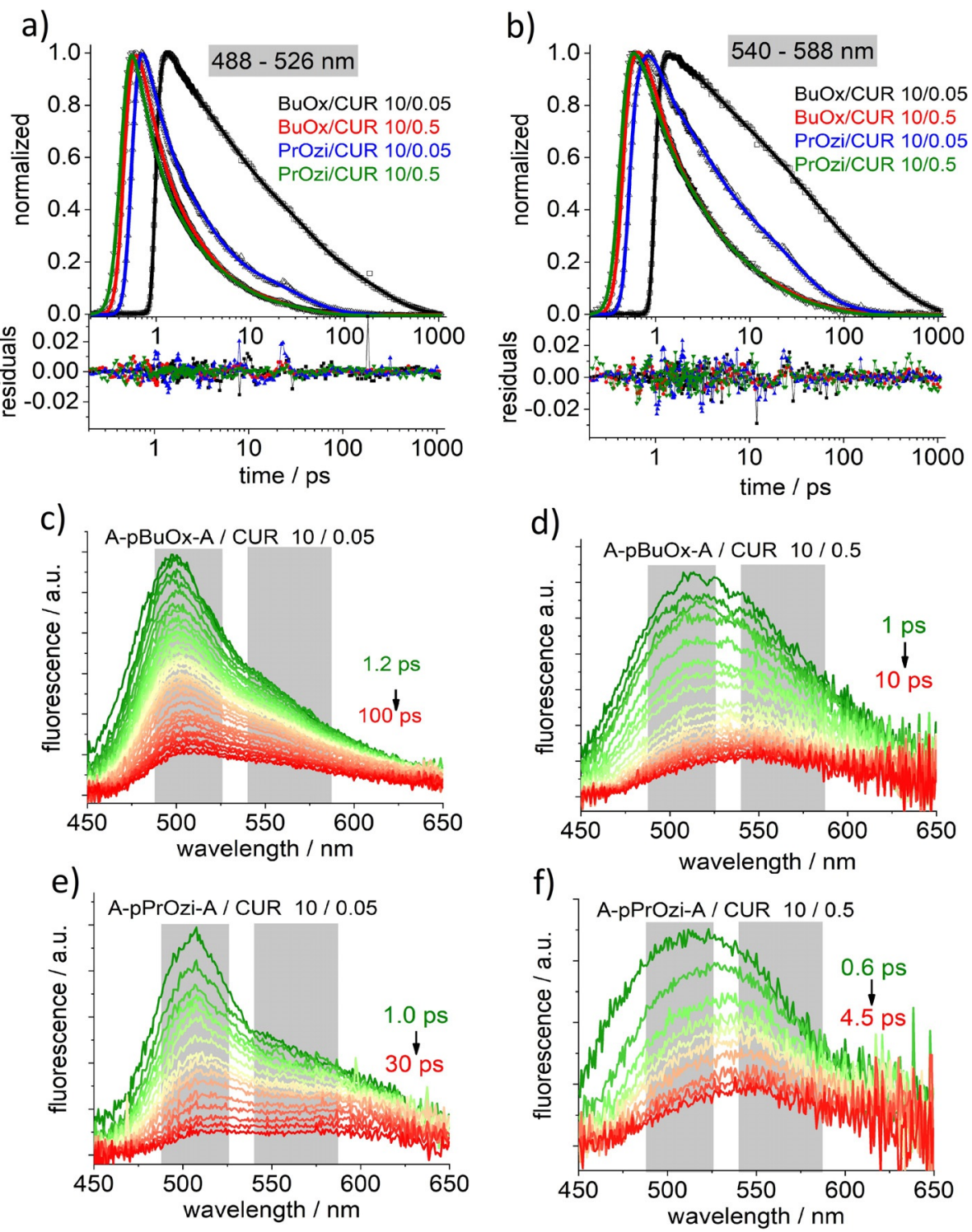

Figure 4. Fluorescence upconversion decays of A-pBuOx-A/CUR $=10 / 0.05$ (black) \& 10/0.5 (red) and A-pPrOzi-A/CUR $=10 / 0.05$ (blue) and 10/0.5 (green) at a) 488-526 nm and b) 540-588 nm. Decays were fitted with a multi-exponential function exhibiting 5 independent lifetimes. Time resolved emission spectra at different delay times of A-pBuOx-A/CUR $=10 / 0.05$ (c) and 10/0.5 (d) and A-pPrOzi-A/CUR=10/0.05 (e) and 10/0.5 (f). Before the measurements, all samples were diluted with $\mathrm{H}_{2} \mathrm{O}$ to a constant CUR concentration of $0.05 \mathrm{~g} \mathrm{~L}^{-1}$ and measured in quartz cuvettes $(d=10 \mathrm{~mm})$.

ed to CUR molecules closely embedded in the polymer matrix of the micellar core.

To get a better understanding about the dynamics of the micellar CUR formulations, dry powder of A-pPrOzi-A or ApBuOx-A was added to aqueous formulations of A-pPrOzi-A/ CUR $=10 / 12$ and A-pBuOx-A/CUR $=10 / 4$, respectively. Therefore, the CUR concentration in all samples was kept essentially constant, whereas the $\mathrm{P} / \mathrm{C}$ ratio increased with increasing amount of polymer added. Both, $\lambda_{\text {abs,max }}$ (Figure $\mathrm{S} 7 \mathrm{a}, \mathrm{d}$ ) as well as $\lambda_{\text {em,max }}$ (Figure $\mathrm{S} 7 \mathrm{~b}, \mathrm{e}$ ) were only dependent on the $\mathrm{P} / \mathrm{C}$ ratio, irrespective if obtained by addition of polymer or by direct formulation. This illustrates the highly dynamic structure of the CUR-loaded micelles (Stokes shifts are shown in Figure S8). Most interestingly, the high-energy emission at $\lambda_{\mathrm{em}}=510 \mathrm{~nm}$ also occurred when additional A-pBuOx-A was added to the
A-pBuOx/CUR $=10 / 4 \mathrm{gL}^{-1}$ formulation (Figure S7e). This was accompanied with a strong increase in fluorescence intensity at high $\mathrm{P} / \mathrm{C}$ ratios as observed for the formulated samples (Figure $3 \mathrm{c}$ ). The increase in emission at high A-pBuOx-A/CUR ratios was also clearly visible after exciting the samples with a conventional UV/Vis lamp at $\lambda_{\mathrm{ex}}=365 \mathrm{~nm}$ (Figure S7 c,f). That the photophysical properties were determined predominantly by the $\mathrm{P} / \mathrm{C}$ ratio was corroborated by comparing formulated samples at different polymer and CUR concentrations, but constant $\mathrm{P} / \mathrm{C}$ ratio (Figure $\mathrm{S} 9$ ). One may argue that such fast exchange dynamics may be detrimental for the envisioned application. That may be the case, in particular if drug targeting is envisioned. However, even though drug targeting using nanoparticles (not antibodies) is a concept that has been heavily investigated for decades, it produced little if any products that 
a)

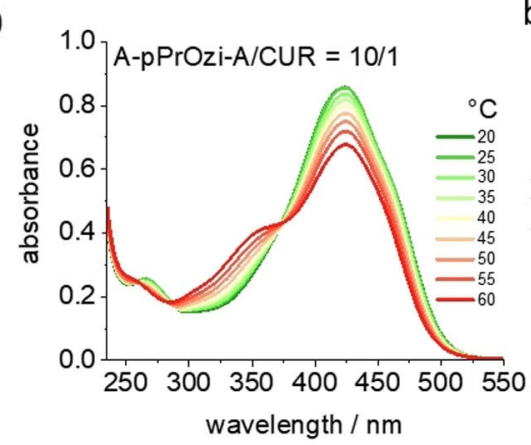

d)

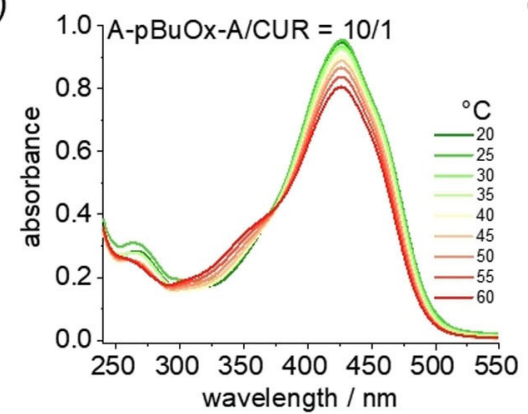

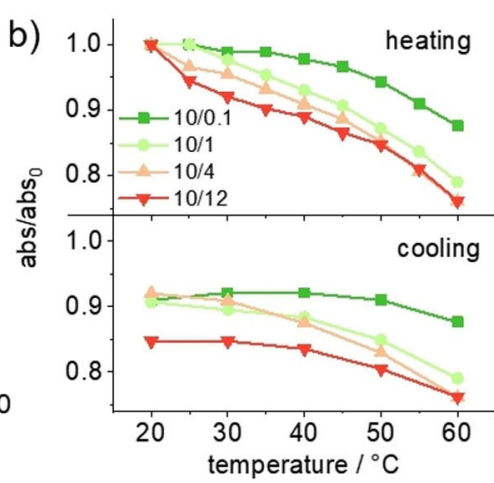

e)

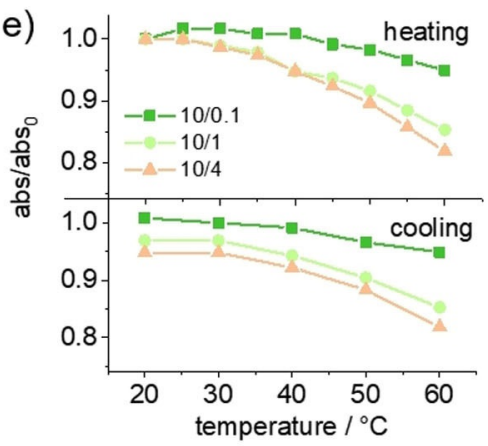

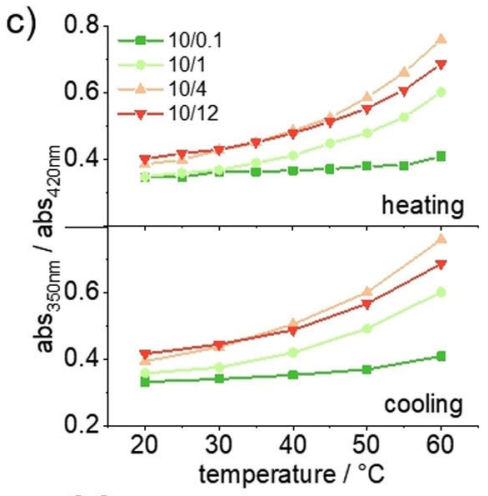

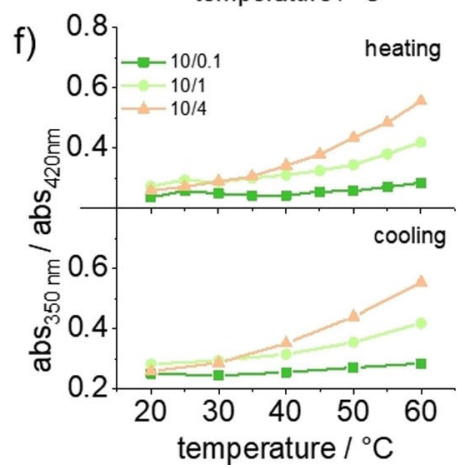

Figure 5. Temperature dependent, normalized absorption spectra of a) $\mathbf{A}-\mathrm{pPrOzi}-\mathrm{A} / \mathrm{CUR}=10 / 1$ and $\mathrm{b}) \mathrm{A}-\mathrm{pBuOx}-\mathbf{A} / \mathrm{CUR}=10 / 1 \mathrm{gL} \mathrm{L}^{-1}$ heated from $20^{\circ} \mathrm{C}$ to

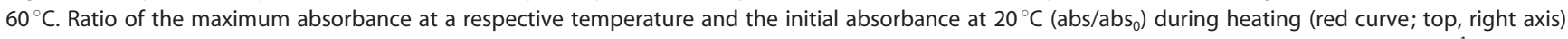
and cooling (blue curve; top, right axis). Ratios of (b,e) abs/abs ${ }_{0}$ and $(c, f) a_{35}{ }_{350 \mathrm{~nm}} / \mathrm{abs}_{420 \mathrm{~nm}}$ for $(\mathrm{b}, \mathrm{c}) \mathrm{A}-\mathrm{pPrOzi}-\mathrm{A} / \mathrm{CUR}=10 / 0.1 ; 10 / 1 ; 10 / 4 ; 10 / 12 \mathrm{gL} \mathrm{L}^{-1}$ and $(\mathrm{e}, \mathrm{f})$ A-pBuOx-A/CUR $=10 / 0.1 ; 10 / 1 ; 10 / 4 \mathrm{gL}^{-1}$ while heating (top curves) and cooling (bottom curves). Prior to the measurements, all samples were diluted with $\mathrm{H}_{2} \mathrm{O}$ to a similar CUR concentration of $\approx 0.01 \mathrm{~g} \mathrm{~L}^{-1}$ and measured in quartz cuvettes with $d=10 \mathrm{~mm}$.

benefit patients, while drug solubilization/formulation is a trusted method that has proven its value time and again, including employing the A-pBuOx-A platform discussed here. ${ }^{[60]}$

Temperature-dependent measurements give additional insights into the stability of the two formulations (Figure 5; for absorbance spectra at different $\mathrm{P} / \mathrm{C}$ ratios, the reader is referred to Figure S10 for A-pPrOzi-A/CUR and Figure S11 for ApBuOx-A/CUR). With increasing temperature, the absorbance at $\lambda_{\text {abs }} \approx 420 \mathrm{~nm}$ decreased and a new absorption at $\lambda_{\text {abs }}=$ $355 \mathrm{~nm}$ occurred, resembling the absorption band of the diketo tautomer of CUR, accompanied by an isosbestic point at $\lambda_{\text {abs }} \approx 370 \mathrm{~nm}$ (Figure $5 \mathrm{a}, \mathrm{d}$ ). The new band became more prominent at elevated temperatures for all samples (Figure $5 c, f)$. The tautomerization led to a distinct change in color (Figure S12) and is more prominent at higher $\mathrm{P} / \mathrm{C}$ ratio (Figure $5 c, f)$. This further corroborates the hypothesis that the intermolecular hydrogen bonds between polymer and CUR weaken with increasing CUR content, due to fewer polymer amide groups available per CUR molecule as well as an increasingly disordered and dynamic situation in the micelles. Moreover, the keto-enol/diketo ratio at a certain CUR concentration and temperature was higher for A-pBuOx-A than for ApPrOzi-A based formulations, also corroborating stronger ApBuOx-A/CUR interactions. Furthermore, in the case of ApBuOx-A, the shift in the tautomeric ratio was completely reversible upon cooling, indicating a higher thermal stability of the A-pBuOx-A/CUR micelles (Figure 5c,f). Most interestingly, for $\mathrm{P} / \mathrm{C}=10 / 0.1$, only a small shoulder occurred at $\lambda_{\text {abs }}$ $\approx 355 \mathrm{~nm}$, irrespective of the polymer structure. Although not detectable in steady state, this fits well to the results obtained from the fluorescence up-conversion studies, in which both polymers exhibited a high-energy emission indicative for strong polymer-CUR interactions only at low CUR content.

Interesting to note, the steady-state fluorescence intensity of A-pPrOzi-A/CUR (Figure S13a,b) as well as A-pBuOx-A/CUR (Figure $\mathrm{S} 14 \mathrm{a}, \mathrm{b}$ ) increased with increasing temperature at low loading $(P / C=10 / 0.1)$. The increase was more pronounced for A-pBuOx-A/CUR (Figure S14). Such increase is unexpected, as the fluorescence quantum yields (and fluorescence intensities) are expected to decrease with increasing temperature (i.e. decreasing viscosity), as internal conversion rates increase with temperature. ${ }^{[40 c]}$ We hypothesize that at such low CUR loadings, water molecules present at low temperature are excluded from the micellar core with increasing temperature, which increases the local viscosity (i.e. decreases mobility) despite the increased temperature. This is supported by the more pronounced increase of the high-energy emission at $\lambda_{\mathrm{em}}=510$ in the case of A-pBuOx-A which is attributed to CUR molecules closely embedded in the polymer matrix of the micellar core. Interestingly at lower $P / C$ ratios, the fluorescence increased only in the case of A-pBuOx-A, whereas with A-pPrOzi-A a decrease in fluorescence intensity occurred. Again, this highlights the highly unusual character of the A-pBuOx-A/CUR nanoformulations. The hypothesis of water exclusion at low $\mathrm{P} / \mathrm{C}$ ratios 
in the case of A-pBuOx-A is further corroborated by the temperature-dependence with respect to the respective micellar morphology. While A-pPrOzi-A based CUR-formulations were more or less unaffected by the heating/cooling cycle (Figure S15a; S16), A-pBuOx-A/CUR exhibited a much stronger, temperature-dependent behavior (Figure S15b,c and S17).

\section{Conclusions}

Curcumin (CUR) is one of the most intensively studied bioactive natural compounds even though its viability as an active pharmaceutical ingredient is heavily debated. ${ }^{[14-15]}$ Key limiting factors to study and use CUR are its low solubility and stability. We studied micellar CUR nanoformulations of the two amphiphiles A-pPrOzi-A and A-pBuOx-A, which are constitutional isomers but exhibit very different CUR loading. While ApPrOzi-A gives access to CUR formulations with drug loadings exceeding 50 wt.\%, A-pBuOx-A micelles cannot be loaded beyond $25 \mathrm{wt} . \%$. Since CUR is solvatochromic, we probed the interactions between CUR and the polymers using spectroscopic methods. With increasing drug loading, the absorbance of CUR showed a profound hypsochromic shift and steadystate fluorescence intensity decreased. At low drug loading, a pronounced emission was observed at $510 \mathrm{~nm}$ in A-pBuOx-A formulations, which was essentially absent in the case of $\mathbf{A}$ pPrOzi-A/CUR. To the best of our knowledge, A-pBuOx-A is the first non-ionic, polymeric system revealing two steadystate emission bands for encapsulated CUR. Fluorescence upconversion experiments revealed that the species responsible for the emission at $510 \mathrm{~nm}$ is in fact present in both micelles, but decays much faster in the case of A-pPrOzi-A. This makes the emission virtually non-observable in steady-state spectroscopy. We attribute this observation to a more restricted molecular mobility of CUR within the hydrophobic core of A-pBuOxA at very low drug loading. In contrast, CUR embedded in ApPrOzi-A micelles retains more flexibility, probably in part due to the higher polymer flexibility. The stronger interactions between CUR and A-pBuOx-A were corroborated by temperature dependent measurements. We find it particularly intriguing that the polymer with the weaker interaction allows much higher drug loading.

Despite the very high loading, the drug loaded micelles exchanged rapidly with free polymer or empty micelles in solution, as was evidenced by the reversal of the hypsochromic shifts in the absorption as well as the appearance of the emission at $510 \mathrm{~nm}$ in the case of A-pBuOx-A.

Even though employing only the model compound CUR in the present contribution, it is clear that similar mechanisms for drug loading and molecular interaction are relevant for many other drug molecules and probably other polymer platforms. Indeed, we have observed similar specificities with respect to drug loading and polymer/drug structure with several other drugs, including different taxanes, ${ }^{[61]}$ antiretroviral efavirenz, ${ }^{[62]}$ and mitotane ${ }^{[63]}$ used in treatment for adrenocortical carcinoma. Our results clearly show that the concept of a hydrophobic polymer that solubilizes a hydrophobic cargo is much too simplistic and a much more detailed look into the interactions be- tween polymer micelle and cargo is necessary. In fact, we provide conclusive evidence that stronger interaction between drug and polymer must not correlate with higher drug loading or the stability of nanoformulations and corroborate that smallest structural changes can significantly affect the interactions between drug and polymer, which must be considered in developing advanced drug nanoformulations.

\section{Acknowledgements}

This work was supported by the Deutsche Forschungsgemeinschaft (Project number 398461692, awarded to R.L.). Moreover, M.M.L. would like to thank the Evonik Foundation for providing a doctoral fellowship. We would also like to thank Christian May for technical support. C.L. is grateful to the Bavarian Ministry of Education, Culture, Research, and the Fine Arts for support within the SolTech consortium. Moreover, we thank Prof. Ann-Christin Pöppler for valuable discussions.

\section{Conflict of interest}

M.M.L. and R.L are listed as inventors on a patent application pertinent to materials discussed in this contribution.

Keywords: curcumin · drug delivery - fluorescence - poly(2oxazine) · poly(2-oxazoline) • polymer-drug interaction upconversion

[1] a) T. Kita, S. Imai, H. Sawada, H. Kumagai, H. Seto, Biosci. Biotechnol. Biochem. 2008, 72, 1789-1798; b) T. Esatbeyoglu, P. Huebbe, I. M. A. Ernst, D. Chin, A. E. Wagner, G. Rimbach, Angew. Chem. Int. Ed. 2012, 51 , 5308-5332; Angew. Chem. 2012, 124, 5402-5427.

[2] S. C. Gupta, S. Patchva, W. Koh, B. B. Aggarwal, Clin. Exp. Pharmacol. Physiol. 2012, 39, 283-299.

[3] T. Ak, i. Gülçin, Chem.-Biol. Interact. 2008, 174, 27-37.

[4] S. Miriyala, M. Panchatcharam, P. Rengarajulu, in The Molecular Targets and Therapeutic Uses of Curcumin in Health and Disease, Springer, 2007, pp. 359-377.

[5] G. M. Cole, B. Teter, S. A. Frautschy, Adv. Exp. Med. Biol. 2007, 595, 197 212.

[6] D.-w. Zhang, M. Fu, S.-H. Gao, J.-L. Liu, Evid.-Based Complementary Altern. Med. 2013, 636053.

[7] J. S. Jurenka, Altern. Med. Rev. 2009, 14, 141-153.

[8] X. Yang, Z. Li, N. Wang, L. Li, L. Song, T. He, L. Sun, Z. Wang, Q. Wu, N. Luo, C. Yi, C. Gong, Sci. Rep. 2015, 5, 10322.

[9] N. Jurrmann, R. Brigelius-Flohé, G.-F. Böl, J. Nutr. 2005, 135, 1859-1864.

[10] J. U. Marquardt, L. Gomez-Quiroz, L. O. Arreguin Camacho, F. Pinna, Y.-H. Lee, M. Kitade, M. P. Domínguez, D. Castven, K. Breuhahn, E. A. Conner, P. R. Galle, J. B. Andersen, V. M. Factor, S. S. Thorgeirsson, J. Hepatol. 2015, 63, 661-669.

[11] a) A. Goel, C. R. Boland, D. P. Chauhan, Cancer Lett. 2001, 172, $111-118$; b) A. L. Clutterbuck, D. Allaway, P. Harris, A. Mobasheri, F1000Research 2013, 2, 147.

[12] a) C. Schneider, O. N. Gordon, R. L. Edwards, P. B. Luis, J. Agric. Food Chem. 2015, 63, 7606-7614; b) O. N. Gordon, P. B. Luis, H. O. Sintim, C. Schneider, J. Biol. Chem. 2015, 290, 4817-4828.

[13] a) L. Shen, H.-F. Ji, Trends Mol. Med. 2012, 18, 138-144; b) K. Lirdprapamongkol, H. Sakurai, S. Suzuki, K. Koizumi, O. Prangsaengtong, A. Viriyaroj, S. Ruchirawat, J. Svasti, I. Saiki, In Vivo 2010, 24, $501-506$.

[14] M. A. W. Jonathan Baell, Nature 2014, 513, 481 - 483.

[15] M. Heger, Nature 2017, 543, 40-40. 
[16] Y. B. Pawar, B. Munjal, S. Arora, M. Karwa, G. Kohli, J. K. Paliwal, A. K Bansal, Pharmaceutics 2012, 4, 517-530.

[17] B. T. Kurien, A. Singh, H. Matsumoto, R. H. Scofield, Assay Drug Dev. Technol. 2007, 5, 567-576.

[18] P. Anand, A. B. Kunnumakkara, R. A. Newman, B. B. Aggarwal, Mol. Pharm. 2007, 4, 807-818.

[19] J. Jeevanandam, Y. S. Chan, M. K. Danquah, Biochimie 2016, 128-129, $99-112$.

[20] a) O. Naksuriya, S. Okonogi, R. M. Schiffelers, W. E. Hennink, Biomaterials 2014, 35, 3365-3383; b) W.-H. Lee, C.-Y. Loo, P. M. Young, D. Traini, R. S Mason, R. Rohanizadeh, Expert Opin. Drug Delivery 2014, 11, 1183-1201; c) S. Datta, A. Jutková, P. Šrámková, L. Lenkavská, V. Huntošová, D Chorvát, P. Miškovský, D. Jancura, J. Kronek, Biomacromolecules 2018, 19, 2459-2471.

[21] S. Lv, Y. Wu, K. Cai, H. He, Y. Li, M. Lan, X. Chen, J. Cheng, L. Yin, J. Am. Chem. Soc. 2018, 140, 1235-1238.

[22] S. Wieczorek, A. Dallmann, Z. Kochovski, H. G. Börner, J. Am. Chem. Soc. 2016, 138, 9349-9352.

[23] a) Y. Li, Q. Zou, C. Yuan, S. Li, R. Xing, X. Yan, Angew. Chem. Int. Ed. 2018 57, 17084-17088; Angew. Chem. 2018, 130, 17330-17334; b) K. Mitra, S. Gautam, P. Kondaiah, A. R. Chakravarty, Angew. Chem. Int. Ed. 2015 54, 13989-13993; Angew. Chem. 2015, 127, 14195-14199.

[24] Y. Shi, M. J. van Steenbergen, E. A. Teunissen, L. S. Novo, S. Gradmann, M. Baldus, C. F. van Nostrum, W. E. Hennink, Biomacromolecules 2013 14, $1826-1837$.

[25] T. Lorson, M. M. Lübtow, E. Wegener, M. S. Haider, S. Borova, D. Nahm, R. Jordan, M. Sokolski-Papkov, A. V. Kabanov, R. Luxenhofer, Biomaterials 2018, 178, 204-280.

[26] a) R. Luxenhofer, A. Schulz, C. Roques, S. Li, T. K. Bronich, E. V. Batrakova, R. Jordan, A. V. Kabanov, Biomaterials 2010, 31, 4972-4979; b) A Schulz, S. Jaksch, R. Schubel, E. Wegener, Z. Di, Y. Han, A. Meister, J. Kressler, A. V. Kabanov, R. Luxenhofer, C. M. Papadakis, R. Jordan, ACS Nano 2014, 8, 2686-2696; c) Z. He, X. Wan, A. Schulz, H. Bludau, M. A Dobrovolskaia, S. T. Stern, S. A. Montgomery, H. Yuan, Z. Li, D. Alakhova, M. Sokolsky, D. B. Darr, C. M. Perou, R. Jordan, R. Luxenhofer, A. V. Kabanov, Biomaterials 2016, 101, 296-309.

[27] M. M. Lübtow, L. Hahn, M. S. Haider, R. Luxenhofer, J. Am. Chem. Soc. 2017, 139, 10980-10983.

[28] M. M. Lübtow, L. C. Nelke, J. Seifert, J. Kühnemundt, G. Sahay, G. Dandekar, S. Nietzer, R. Luxenhofer, J. Controlled Release 2019, 303, 162-180

[29] S. Jaksch, A. Schulz, Z. Di, R. Luxenhofer, R. Jordan, C. M. Papadakis, Macromol. Chem. Phys. 2016, 217, 1448-1456.

[30] a) M. M. Lübtow, L. Keßler, A. Appelt-Menzel, T. Lorson, N. Gangloff, M. Kirsch, S. Dahms, R. Luxenhofer, Macromol. Biosci. 2018, 18, 1800155; b) L. Hahn, M. M. Lübtow, T. Lorson, F. Schmitt, A. Appelt-Menzel, R. Schobert, R. Luxenhofer, Biomacromolecules 2018, 19, 3119-3128.

[31] a) C. Párkányi, M. R. Stem-Beren, O. R. Martínez, J.-J. Aaron, M. Bulaceanu-MacNair, A. F. Arrieta, Spectrochim. Acta Part A 2004, 60, 1805-1810; b) S. N. Margar, N. Sekar, Mol. Phys. 2016, 114, 1867-1879.

[32] K. I. Priyadarsini, J. Photochem. Photobiol. C 2009, 10, 81 -95.

[33] D. Patra, C. Barakat, Spectrochim. Acta Part A 2011, 79, 1034-1041.

[34] a) T. M. Kolev, E. A. Velcheva, B. A. Stamboliyska, M. Spiteller, Int. J. Quantum Chem. 2005, 102, 1069-1079; b) E. Benassi, F. Spagnolo, Theor Chem. Acc. 2009, 124, 235-250.

[35] A. Dutta, B. Boruah, A. K. Manna, B. Gohain, P. M. Saikia, R. K. Dutta, Spectrochim. Acta Part A 2013, 104, 150-157.

[36] C. Banerjee, S. Ghosh, S. Mandal, J. Kuchlyan, N. Kundu, N. Sarkar, J. Phys. Chem. B 2014, 118, 3669-3681.

[37] S. Ghosh, J. Kuchlyan, D. Banik, N. Kundu, A. Roy, C. Banerjee, N. Sarkar, J. Phys. Chem. B 2014, 118, 11437-11448.

[38] M. Ghosh, A. T. K. Singh, W. Xu, T. Sulchek, L. I. Gordon, R. O. Ryan, Nanomedicine 2011, 7, 162-167.

[39] a) R. Adhikary, P. J. Carlson, T. W. Kee, J. W. Petrich, J. Phys. Chem. B 2010, 114, 2997-3004; b) C. Banerjee, S. Maiti, M. Mustafi, J. Kuchlyan, D. Banik, N. Kundu, D. Dhara, N. Sarkar, Langmuir 2014, 30, 10834 10844.
[40] a) G. Oster, Y. Nishijima, J. Am. Chem. Soc. 1956, 78, 1581-1584; b) S. Sharafy, K. A. Muszkat, J. Am. Chem. Soc. 1971, 93, 4119-4125; c) T. Förster, G. Hoffman, Z. Phys. Chem. 1971, 75, 63-76; d) G. C. Schmidt, Annalen der Physik 1896, 294, 103-130; e) G.C. Schmidt, Annalen der Physik 1921, 370, 247-256; f) J. Stark, F. Lipp, Z. Phys. Chem. 1914, 86U, $36-50$.

[41] J. Zuegg, M. A. Cooper, Curr. Top. Med. Chem. 2012, 12, 1500-1513.

[42] Y. Manolova, V. Deneva, L. Antonov, E. Drakalska, D. Momekova, N. Lambov, Spectrochim. Acta Part A 2014, 132, 815-820.

[43] Z. Moussa, M. Chebl, D. Patra, J. Photochem. Photobiol. B 2017, 173, $307-317$.

[44] M. Bernabé-Pineda, M. T. Ramírez-Silva, M. Romero-Romo, E. González Vergara, A. Rojas-Hernández, Spectrochim. Acta Part A 2004, 60, 1091 1097.

[45] R. Ghosh, J. A. Mondal, D. K. Palit, J. Phys. Chem. B 2010, 114, 1212912143.

[46] K. Muthoosamy, I. B. Abubakar, R. G. Bai, H.-S. Loh, S. Manickam, Sci. Rep. 2016, 6, 32808.

[47] I. O. Omotuyi, M. O. Abiodun, K. Komolafe, O. C. Ejelonu, O. Olusanya, J. Mol. Model. 2015, 21, 109.

[48] R. Adhikary, P. Mukherjee, T. W. Kee, J. W. Petrich, J. Phys. Chem. B 2009, 113, 5255-5261.

[49] M. Gerecke, G. Bierhance, M. Gutmann, N. P. Ernsting, A. Rosspeintner, Rev. Sci. Instrum. 2016, 87, 053115.

[50] D. Ke, X. Wang, Q. Yang, Y. Niu, S. Chai, Z. Chen, X. An, W. Shen, Langmuir 2011, 27, 14112-14117.

[51] Y. J. Wang, M. H. Pan, A. L. Cheng, L. I. Lin, Y. S. Ho, C. Y. Hsieh, J. K. Lin, J. Pharm. Biomed. Anal. 1997, 15, 1867-1876.

[52] R. Waranyoupalin, S. Wongnawa, M. Wongnawa, C. Pakawatchai, P. Panichayupakaranant, P. Sherdshoopongse, Cent. Eur. J. Chem. 2009, 7, $388-$ 394.

[53] S. M. Khopde, K. Indira Priyadarsini, D. K. Palit, T. Mukherjee, Photochem. Photobiol. 2007, 72, 625-631.

[54] a) J. Zhao, S. Ji, Y. Chen, H. Guo, P. Yang, Phys. Chem. Chem. Phys. 2012, 14, 8803-8817; b) A. S. Klymchenko, A.P. Demchenko, New J. Chem. 2004, 28, 687-692.

[55] M. Mouslmani, D. Patra, RSC Adv. 2014, 4, 8317.

[56] C. Banerjee, C. Ghatak, S. Mandal, S. Ghosh, J. Kuchlyan, N. Sarkar, J. Phys. Chem. B 2013, 117, 6906-6916.

[57] G. Morgese, B. Verbraeken, S. N. Ramakrishna, Y. Gombert, E. Cavalli, J.G. Rosenboom, M. Zenobi-Wong, N. D. Spencer, R. Hoogenboom, E. M. Benetti, Angew. Chem. Int. Ed. 2018, 57, 11667-11672; Angew. Chem. 2018, 130, $11841-11846$.

[58] a) L. Nardo, R. Paderno, A. Andreoni, M. Másson, T. Haukvik, H. H. Tønnesen, Spectroscopy 2008, 22, 187-198; b) S. M. Khopde, K. Indira Priyadarsini, D. K. Palit, T. Mukherjee, Photochem. Photobiol. 2000, 72, 625631.

[59] A. Sillen, Y. Engelborghs, Photochem. Photobiol. 1998, 67, 475-486.

[60] a) Z. He, A. Schulz, X. Wan, J. Seitz, H. Bludau, D. Y. Alakhova, D. B. Darr, C. M. Perou, R. Jordan, I. Ojima, A. V. Kabanov, R. Luxenhofer, J. Controlled Release 2015, 208, 67-75; b) X. Wan, Y. Min, H. Bludau, A. Keith, S. S. Sheiko, R. Jordan, A. Z. Wang, M. Sokolsky-Papkov, A. V. Kabanov, ACS Nano 2018, 12, 2426-2439; c) X. Wan, J. J. Beaudoin, N. Vinod, Y. Min, N. Makita, H. Bludau, R. Jordan, A. Wang, M. Sokolsky, A. V. Kabanov, Biomaterials 2019, 192, 1-14.

[61] Y. Seo, A. Schulz, Y. Han, Z. He, H. Bludau, X. Wan, J. Tong, T. K. Bronich, M. Sokolsky, R. Luxenhofer, R. Jordan, A. V. Kabanov, Polym. Adv. Technol. 2015, 26, 837-850.

[62] M. M. Lübtow, M. S. Haider, M. Kirsch, S. Klisch, R. Luxenhofer, Biomacromolecules 2019, 20, $3041-3056$

[63] M. S. Haider, J. Schreiner, S. Kendl, M. Kroiß, R. Luxenhofer, ChemRxiv 2019, https://doi.org/10.26434/chemrxiv.8055773.v1.

Manuscript received: June 7, 2019

Accepted manuscript online: July 10, 2019

Version of record online: September 2, 2019 